\title{
Incremental value of left atrial booster and reservoir strain in predicting atrial fibrillation in patients with hypertrophic cardiomyopathy: a cardiovascular magnetic resonance study
}

Betty Raman ${ }^{1 *} \mathbb{D}$, Robert W. Smillie ${ }^{1 \dagger}$, Masliza Mahmod ${ }^{1}$, Kenneth Chan ${ }^{1}$, Rina Ariga', Chrysovalantou Nikolaidou', Elizabeth Ormondroyd², Kate Thomson², Andrew R. Harper², Gifford Tan', Adam J. Lewandowski ${ }^{2}$, Fernando Rodriguez Bajo ${ }^{1}$, Eleanor C. Wicks ${ }^{1}$, Barbara Casadei ${ }^{2}$, Hugh Watkins ${ }^{2 \dagger}$ and Stefan Neubauer ${ }^{1+}$

\begin{abstract}
Background: Left atrial (LA) size and function are known predictors of new onset atrial fibrillation (AF) in hypertrophic cardiomyopathy (HCM) patients. Components of LA deformation including reservoir, conduit, and booster function provide additional information on atrial mechanics. Whether or not LA deformation can augment our ability to predict the risk of new onset AF in HCM patients beyond standard measurements is unknown.

Methods: We assessed LA size, function, and deformation on cardiovascular magnetic resonance (CMR) in 238 genotyped HCM patients and compared this with twenty age, sex, blood pressure and body mass index matched control subjects. We further evaluated the determinants of new onset AF in HCM patients.

Results: Compared to control subjects, HCM patients had higher LA antero-posterior diameter, lower LA ejection fraction and lower $L A$ reservoir $(19.9[17.1,22.2], 21.6[19.9,22.9], P=0.047)$ and conduit strain $(10.6 \pm 4.4,13.7 \pm 3.3$, $P=0.002$ ). LA booster strain did not differ between healthy controls and HCM patients, but HCM patients who developed new onset AF $(n=33)$ had lower booster strain $(7.6 \pm 3.3,9.5 \pm 3.0, P=0.001)$ than those that did not $(n=205)$. In separate multivariate models, age, LA ejection fraction, and LA booster and reservoir strain were each independent determinants of AF. Age $\geq 55$ years was the strongest determinant ( $\mathrm{HR} 6.62,95 \% \mathrm{Cl}$ 2.79-15.70), followed by LA booster strain $\leq 8 \%(H R 3.69,95 \% \mathrm{Cl} 1.81-7.52)$ and LA reservoir strain $\leq 18 \%$ (HR 2.56, 95\% Cl 1.24-5.27). Conventional markers of HCM phenotypic severity, age and sudden death risk factors were associated with LA strain components.
\end{abstract}

\footnotetext{
${ }^{*}$ Correspondence: betty.raman@cardiov.ox.ac.uk

${ }^{\dagger}$ Betty Raman and Robert W. Smillie-joint first authors

${ }^{\dagger}$ Hugh Watkins and Stefan Neubauer-joint senior authors

${ }^{1}$ University of Oxford Centre for Clinical Magnetic Resonance Research

(OCMR), Division of Cardiovascular Medicine, Radcliffe Department

of Medicine, University of Oxford, Oxfordshire OX3 9DU, United Kingdom

Full list of author information is available at the end of the article
}

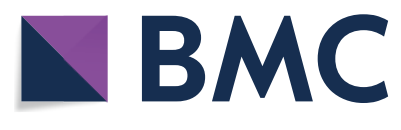

(c) The Author(s) 2021. Open Access This article is licensed under a Creative Commons Attribution 4.0 International License, which permits use, sharing, adaptation, distribution and reproduction in any medium or format, as long as you give appropriate credit to the original author(s) and the source, provide a link to the Creative Commons licence, and indicate if changes were made. The images or other third party material in this article are included in the article's Creative Commons licence, unless indicated otherwise in a credit line to the material. If material is not included in the article's Creative Commons licence and your intended use is not permitted by statutory regulation or exceeds the permitted use, you will need to obtain permission directly from the copyright holder. To view a copy of this licence, visit http://creativecommons.org/licenses/by/4.0/. The Creative Commons Public Domain Dedication waiver (http://creativeco mmons.org/publicdomain/zero/1.0/) applies to the data made available in this article, unless otherwise stated in a credit line to the data. 
Conclusions: LA strain components are impaired in HCM and, together with age, independently predicted the risk of new onset AF. Increasing age and phenotypic severity were associated with LA strain abnormalities. Our findings suggest that the routine assessment of LA strain components and consideration of age could augment LA size in predicting risk of $\mathrm{AF}$, and potentially guide prophylactic anticoagulation use in $\mathrm{HCM}$.

Keywords: Hypertrophic cardiomyopathy, Atrial fibrillation, Cardiovascular magnetic resonance imaging, Left atrial strain, Booster strain, Reservoir strain

\section{Background}

At least 1 in 5 patients with hypertrophic cardiomyopathy (HCM) are affected by atrial fibrillation (AF) [1], the development of which heralds an unfavourable prognosis and is associated with a significantly higher all-cause mortality due to an increased risk of heart failure and stroke [1]. Current guidelines for HCM recommend a 48-h ambulatory Holter monitor every 2 years [2], though this may be inadequate for detecting new onset AF [3]. Lifelong anti-coagulation is recommended once AF is detected as cardioembolic risk is especially high in HCM. However, given the limitations of intermittent monitoring, anticoagulation sometimes follows an embolic complication. Therefore, the initiation of anti-coagulation therapy is clearly desirable when AF is anticipated.

Previous work has identified predictors for new onset AF in HCM. These include age [4], left atrial (LA) diameter [5], indexed LA end-diastolic volume (LAEDV) [6], and more recently, LA function [7]. However, contemporary guidelines have been slow to incorporate these biomarkers into the routine clinical management of HCM patients. Currently, the American Heart Association (AHA) recommends Holter monitoring for AF if a patient complains of palpitations [8]. The European Society of Cardiology (ESC) recommends intensification of arrhythmia surveillance when the anterior-posterior diameter of the LA exceeds $45 \mathrm{~mm}$ on echocardiography [2]. Specific recommendations for prophylactic anticoagulation are lacking and clinicians may initiate therapy once atrial diameter exceeds a given size in anticipation of AF. However, studies are emerging that show previously reported thresholds for LA size may be too conservative; with $59 \%$ of AF cases in one study occurring in patients with LA diameter $<45 \mathrm{~mm}$ [7]. More refined markers that determine AF risk are therefore clearly required.

The role of LA volume and diameter in predicting AF onset in HCM is well established. However, studies assessing the link between LA function and AF risk are limited. Determinants of LA function in HCM are complex and may include direct, myopathic aspects (where the mutant sarcomeric protein is expressed in atrial muscle) as well as secondary hemodynamic forces relating to increased atrial pressures in outflow obstruction, mitral regurgitation and diastolic dysfunction $[9,10]$. LA function can be assessed using LA ejection fraction (LAEF) and/or LA deformation analysis on 2-dimensional (2D) cardiovascular magnetic resonance (CMR) feature tracking such as LA strain. LA strain consists of reservoir, conduit, and booster components. Whilst LA conduit strain is derived from the motion of atrial tissue during passive ventricular filling, LA reservoir strain and booster strain reflect passive and active LA functions, respectively [11, 12]. Comprehensive studies in HCM patients examining the association between AF and LA reservoir, conduit, and booster strain parameters on (CMR are lacking, yet LA strain components are emerging as sensitive markers for detecting impairment in LA function [13-17].

In this work, we set out to test the hypothesis that LA reservoir and booster strain on CMR will improve our ability to predict risk of incident AF. We further examined the determinants of LA strain components in HCM patients.

\section{Methods \\ Study population and protocol}

This is a retrospective analysis of data from an observational study approved by a local ethics committee (Reference: 07/Q1607/66, 12/LO/ 1979). Patients were recruited from the Inherited Cardiac Conditions (ICC) study at the University of Oxford between 2003 and 2016. As per the ESC diagnostic criteria, the recruitment for this study captured patients with genetically diagnosed or familial HCM who had wall thickness $\geq 13 \mathrm{~mm}$ [2], or non-familial HCM patients with wall thickness $\geq 15 \mathrm{~mm}$ but no other cause of hypertrophy identified. Genetic diagnosis used a minimum of 13-gene testing and patients were stratified based on genotype status (see Additional file 1 for details). Baseline characteristics including symptoms, comorbidities, and medications were retrieved from clinic letters. Patients with any history of AF at the time of CMR were excluded from this study. This exclusion was implemented after reviewing clinic letters and the results of a 24-48 h Holter carried out on each patient. Patients taking amiodarone were excluded. Patients with coronary artery disease, moderate to severe aortic stenosis, HCM phenocopies 


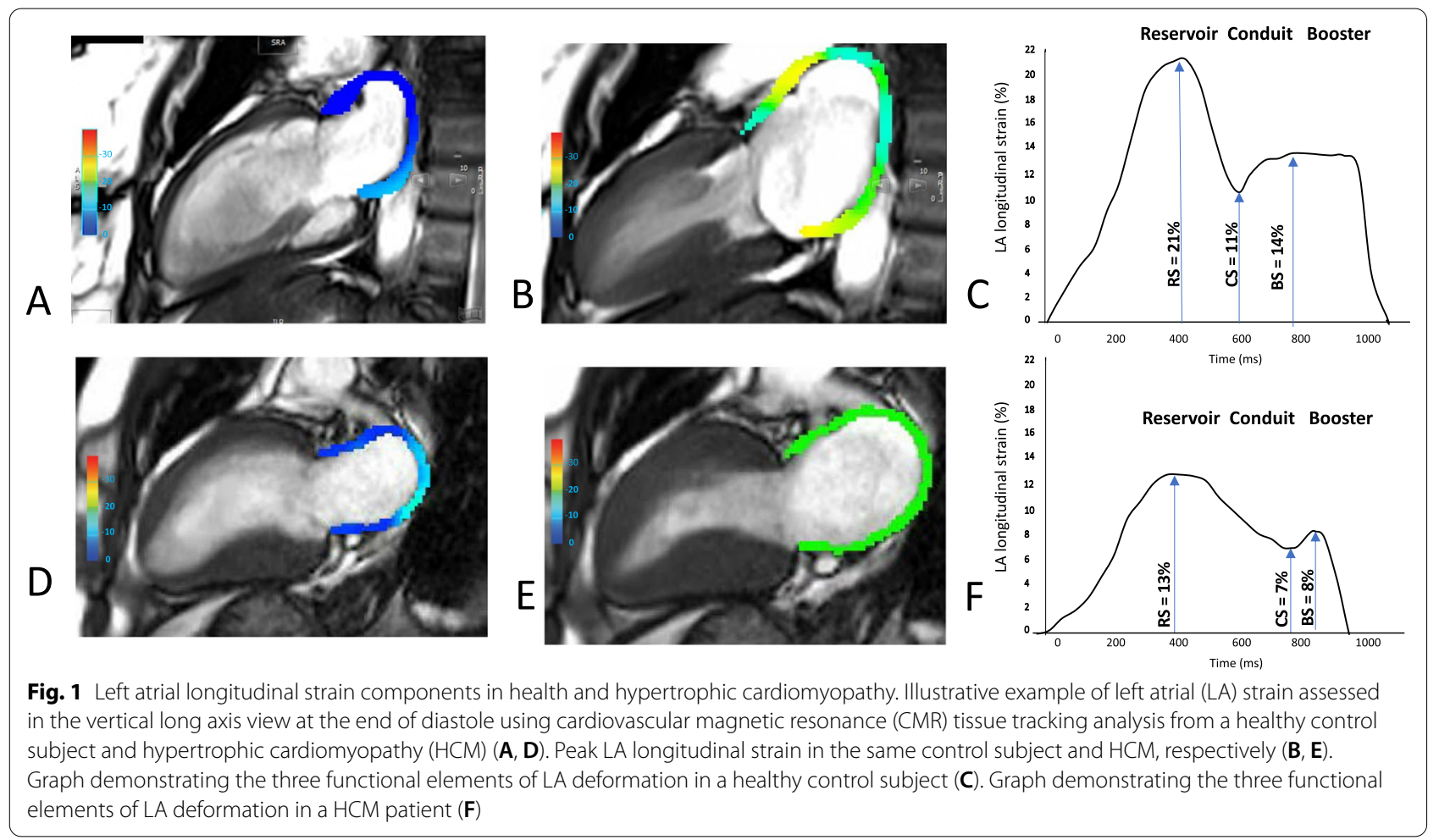

(amyloidosis, Fabry diseases, Danon disease), or contraindications to CMR were also excluded.

Twenty age, sex, blood pressure and body mass index (BMI) matched healthy subjects from another ethically approved study [18] provided normal values in light of potential variability in published normal ranges arising from inter-vendor strain differences. These healthy controls were free of known cardiovascular disease or family history of cardiac disease and were screened for AF on a 12-lead electrocardiogram (ECG). LA data (size, function, and strain) from age, sex and BMI matched controls were also specifically included to define the extent of impairment in LA strain components between HCM and non-HCM subjects.

\section{Cardiovascular magnetic resonance}

CMR image acquisition including cine and late gadolinium enhancement (LGE) used established methods (see Additional file 1 for details). cvi42 software (Circle Cardiovascular Imaging Inc, Calgary, Alberta, Canada) was used as per established methods to assess left ventricular (LV) volume, function, mass and wall thickness and LA anterior-posterior diameter in the LV outflow tract (LVOT) view [19]. As previously described, LAEDV index, indexed LA end-systolic volume (LAESV), and LAEF were assessed from the horizontal and vertical long axis views [20,21]. Similarly, LA peak longitudinal reservoir, conduit and booster strain were assessed using an automated tracking algorithm [22] (see Additional file 1 for details). 2-D feature-tracking strain analysis was averaged across the horizontal and vertical long axis views to derive strain components from cine images. Figure 1 depicts the aspect of this derivation from the vertical long axis. The LA image analysis was performed by an observer (RS) blinded to the clinical information or outcomes. LGE analysis was undertaken by setting the signal intensity threshold at six standard deviations above the mean intensity of a reference region of myocardium that had no visual evidence of enhancement [20].

\section{Clinical follow up}

Patients in the Inherited Cardiac Conditions study, from which this study recruited, were followed up on a yearly basis either in clinic, or with a phone interview, and review of medical records and clinical letters if not followed-up in Oxford. The median follow-up time of those in this study was of 4.5 years. Follow-up was in line with clinical practice and consisted of a clinical visit and assessment, a 12-lead ECG, and 24-48 h Holter (2-yearly). The primary endpoint for this study was new onset AF, defined as an irregularly irregular heart rhythm with absent P-waves documented on a 12-lead ECG or 24-48 h Holter. 


\section{Statistical analysis}

Statistical analysis utilised R (version 3.5.0, R Foundation for Statistical Computing, Vienna, Austria). Packages used were: Survival, pROC, and time ROC. Normality of data was assessed by the Shapiro-Wilk and Anderson-Darling tests, visual inspection of quantile-quantile plots, and consideration for skew and kurtosis values. Mean (standard deviation/SD) and Median [Interquartile range/IQR] are presented accordingly for normally- and non-normally distributed data, respectively. Independent $t$-tests and Mann-Whitney tests were used for normally- and non-normally distributed data, respectively. Categorical variables are summarised as proportions (\%) and the $x^{2}$ (with continuity correction) or Fisher's exact tests were used to compare proportions. Inter- and intra-observer variability of strain measurements was determined by the interclass correlation coefficient, and coefficient of variation.

Stratification of the main LA parameters was based on the optimal thresholds (cut-off values) of these variables for predicting new onset AF, as calculated using Youden's index in conjunction with standard Receiver Operator Characteristic (ROC) analysis. For clinical applicability we rounded these thresholds.

Time-dependent ROC estimations were used to calculate $\mathrm{C}$-statistics (concordance statistics), equivalent to the area under a ROC curve (AUC) and representative of the accuracy of a single value in the diagnosis of new onset AF at 3 years.

Univariate Cox proportional hazard regression analysis was performed to identify predictors of new onset AF. Relative risks were presented as hazard ratios (HR) with 95\% confidence intervals (CI). Where appropriate, the proportional hazards assumption, presence of outliers, and linearity were tested for. Parameters under a significance threshold of $P<0.05$ were included in the Cox multiple regression analysis. Kaplan-Meier cumulative survival curves free of AF were constructed for LA reservoir and booster strain values stratified by the rounded cut-offs. The survival curves were compared with the log-rank test. Simple linear and multiple regression was used to determine the variables that associate with LA strain. Unless adjustments were made for multiple tests, a p-value of $P<0.05$ was considered significant throughout, and all tests were 2 -sided.

The Bonferroni correction was applied selectively to the analysis in Table 1 and to the parameters included in the univariate Cox proportional hazard regression analysis to adjust 'family-wise' error rates in multiple comparisons of related groups and reduce the risk of type I error, whilst acknowledging the conservative nature of the correction. Correction was applied to the parameters relating to the LA (10 parameters in Table 1 , giving an adjusted $P$ of $0.05 / 10=P<0.005$, and 15 parameters in the univariate analysis, giving an adjusted $P$ of $0.05 / 15=P<0.0034)$, and separately to those relating to the $\mathrm{LV}$ (7 parameters in both analyses, giving an adjusted $P$ of $P<0.0071)$. In the univariate analysis correction was also applied to the remaining baseline, clinical and medication parameters (28 parameters giving an adjusted $P$ of $P<0.0018$ ). By contrast the Bonferroni correction was not applied to the simple linear regression for the determinants of LA strain. This decision was made as this aspect of the study involved post-hoc testing of unplanned comparisons that we deem as hypotheses for further exploratory investigation and so avoiding a type II error was of greater importance [23].

\section{Results}

\section{Reproducibility of LA strain measurements}

Inter-observer (GT) and intra-observer variability of LA strain were within the clinically acceptable range (refer to Additional file 1 and Table S1 for details).

\section{Baseline characteristics of HCM patients and controls}

Of the 281 patients screened for study inclusion, 24 (9\%) were excluded due to pre-existing AF at the time of the CMR scan, 14 (5\%) were excluded due to taking amiodarone, and $5(2 \%)$ were excluded due to issues with data collection, leaving $238 \mathrm{HCM}$ patients in the study (see Additional file 1: Fig. S1).

The median subject age was 54 years with IQR $[43,63])$, 184 (77\%) were men, 107 (47\%) were on beta blockers, the average 5-year risk of sudden cardiac death (SCD) was low based on the ESC guidelines for risk stratification $(2.0 \pm 1.6 \%), 69$ (29\%) had mitral regurgitation $(81 \%$ of which was mild or moderate), and 9 (4\%) had LVOT obstruction $\geq 30 \mathrm{mmHg}$. Thirty-three (14\%) developed new onset AF during follow-up after a mean time of 3.6 years (median of 3.0 years with IQR $[2.8,6.8]$ ). Of these, 11 were in persistent AF whereas 22 had evidence of paroxysmal AF. The baseline clinical characteristics of patients and healthy controls are summarised in Table 1.

As expected, when compared to controls, HCM patients had smaller indexed LV end-diastolic and endsystolic volumes, and higher LV ejection fraction (LVEF) and LV mass (Table 1), although after application of the Bonferroni correction only LV mass differed significantly. LA diameter in the LVOT view and LAESV index were higher in HCM than controls, whilst LAEF, reservoir and conduit strain were lower in HCM (Fig. 1) although of these findings, only those relating to conduit strain and LAEF were consistent after the Bonferroni correction was applied. There was a trend towards LAEDV index being increased in HCM, whereas consistent with a previous study, LA booster strain did not differ between 
Table 1 Baseline and CMR data: 'controls vs HCM'; 'No new onset AF vs new onset AF'

\begin{tabular}{|c|c|c|c|c|c|c|}
\hline & $\begin{array}{l}\text { Control subjects } \\
(n=20)\end{array}$ & $\begin{array}{l}\text { HCM population, } \\
n=238\end{array}$ & $P$ value & New onset AF ( $n=33)$ & $\begin{array}{l}\text { No new onset AF } \\
(n=205)\end{array}$ & $P$ value \\
\hline \multicolumn{7}{|l|}{ Baseline and clinical } \\
\hline Age at CMR scan & $48[37,64]$ & $54[43,63]$ & 0.362 & $61[55,66]$ & $53[41,61]$ & $0.001^{*}$ \\
\hline Men, $n, \%$ & 15,75 & 184,77 & 1 & 25,76 & 159,78 & 0.824 \\
\hline Body mass index & $26.6[23.4,27.8]$ & $27.5[24.6,30.3]$ & 0.145 & $28.5[25.7,30.8]$ & $27.2[24.5,30.7]$ & 0.129 \\
\hline Body surface area & $1.9(0.2)$ & $2.0(0.3)$ & 0.276 & $2.1[1.8,2.2]$ & $2.0[1.8,2.2]$ & 0.51 \\
\hline $\begin{array}{l}\text { Systolic blood pressure } \\
(\mathrm{mmHg})\end{array}$ & $128(13.3)$ & $131(18.5)$ & 0.558 & $130(14.8)$ & $131(19.2)$ & 0.968 \\
\hline $\begin{array}{l}\text { Diastolic blood pressure } \\
(\mathrm{mmHg})\end{array}$ & $72(8.0)$ & $77(10.3)$ & 0.077 & $78(9.7)$ & $77(10.4)$ & 0.586 \\
\hline Hypertension, n, \% & 4,20 & 99,42 & 0.479 & 18,55 & 81,40 & 0.130 \\
\hline $\begin{array}{l}\text { Mitral regurgitation } \\
\text { class, \%: no mitral regur- } \\
\text { gitation, } 1,2,3,4\end{array}$ & $0,0,0,0,0$ & $71,17,7,6,0$ & - & $73,3,15,9,0$ & $71,19,5,5,0$ & $0.014^{*}$ \\
\hline $\begin{array}{l}\text { SCD score: ESC guide- } \\
\text { lines }\end{array}$ & N/A & $2.0(1.6)$ & - & $2.3(1.8)$ & $2.0(1.4)$ & 0.273 \\
\hline VT historical, n, \% & N/A & 12,5 & - & 5,15 & 7,3 & $0.015^{*}$ \\
\hline VT new onset, n, \% & N/A & 26,11 & - & 6,18 & 20,10 & 0.225 \\
\hline TIA historical, n, \% & N/A & 6,3 & - & 1,3 & 5,2 & 0.596 \\
\hline TIA new onset, n, \% & 0,0 & 5,2 & - & 2,6 & 3,1 & 0.143 \\
\hline Diabetes mellitus, n, \% & 0,0 & 18,8 & - & 2,6 & 16,8 & 1 \\
\hline Smoking history, n, \% & - & 59,25 & - & 10,30 & 49,24 & 0.516 \\
\hline LGE presence, n, \% & 0,0 & 185,77 & - & 30,91 & 155,76 & 0.056 \\
\hline LGE mass (g) & $0,0 \%$ & $15.0[8.0,26.0]$ & - & $14.0[6.9,29.0]$ & $16.0[8.0,26.0]$ & 0.687 \\
\hline LGE (\%) & $0,0 \%$ & $10.5[5.6,15.5]$ & - & $10.0[4.8,14.6]$ & $10.7[5.7,15.5]$ & 0.601 \\
\hline $\begin{array}{l}\text { Latest NYHA class, \%: } 1 \text {, } \\
2,3,4\end{array}$ & $20,0,0,0$ & $65,28,7,0$ & - & $44,50,6,0$ & $69,25,7,0$ & $0.012^{*}$ \\
\hline $\begin{array}{l}\text { Sarcomeric variant } \\
\text { present, } n, \%\end{array}$ & N/A & 78,33 & - & 8,24 & 70,34 & 0.239 \\
\hline \multicolumn{7}{|l|}{ Medications } \\
\hline Beta-blockers, n, \% & 0,0 & 107,45 & $<0.001^{*}$ & 19,58 & 88,43 & 0.190 \\
\hline $\begin{array}{l}\text { Calcium channel block- } \\
\text { ers, } n, \%\end{array}$ & 0,0 & 59,25 & 0.021 & 12,36 & 47,23 & 0.136 \\
\hline ACE-I/ARB, $n, \%$ & 2,10 & 57,24 & 0.213 & 11,33 & 46,22 & 0.194 \\
\hline Diuretics, n, \% & 0,0 & 9,4 & 0.754 & 1,3 & 8,4 & 1 \\
\hline Aspirin, n, \% & 1,5 & 81,34 & $0.009^{*}$ & 11,33 & 70,34 & 0.845 \\
\hline $\begin{array}{l}\text { Warfarin or other antico- } \\
\text { agulation, n, } \%\end{array}$ & 0,0 & 21,9 & 0.315 & 11,33 & 10,5 & $<0.001^{*}$ \\
\hline \multicolumn{7}{|l|}{ CMR: left atrium } \\
\hline LA diameter (mm) & $34.5(4.3)$ & $37.2(5.7)$ & 0.037 & $39.0[34.0,42.0]$ & $37.0[33.0,40.0]$ & 0.136 \\
\hline LAEDV index $\left(\mathrm{mL} / \mathrm{m}^{2}\right)$ & $38.1[35.9,46.9]$ & $43.8[36.2,51.1]$ & 0.158 & $45.6[39.4,65.5]$ & $43.5[35.2,50.7]$ & 0.044 \\
\hline LAEDV $(\mathrm{mL})$ & $78.7(20.4)$ & $90.8(31.7)$ & 0.095 & $95.3[77.7,127.9]$ & $84.5[66.2,105.7]$ & 0.040 \\
\hline LAESV index $\left(\mathrm{mL} / \mathrm{m}^{2}\right)$ & $17.3(4.7)$ & $23.6(12.4)$ & 0.025 & $31.5(18.3)$ & $22.3(10.7)$ & $<0.001^{*}$ \\
\hline LAESV $(\mathrm{mL})$ & $33.5(10.3)$ & $46.9(24.8)$ & 0.017 & $62.9(36.5)$ & $44.28(21.3)$ & $<0.001^{*}$ \\
\hline LA stroke volume (mL) & $45.2(12.9)$ & $44.0(15.2)$ & 0.720 & $40.0(16.9)$ & $44.6(14.9)$ & 0.111 \\
\hline LA reservoir strain (\%) & $21.6[19.9,22.9]$ & $19.9[17.1,22.2]$ & 0.047 & $15.6(5.4)$ & $19.8(4.3)$ & $<0.001^{*}$ \\
\hline LA conduit strain (\%) & $13.7(3.3)$ & $10.6(4.4)$ & $0.002^{*}$ & $8.6(3.8)$ & $10.9(4.4)$ & $0.004^{*}$ \\
\hline LA booster strain (\%) & $8.7(1.8)$ & $9.2(3.1)$ & 0.439 & $7.6(3.3)$ & $9.5(3.0)$ & $0.001^{*}$ \\
\hline LAEF (\%) & $57.2[54.6,61.5]$ & $51.7[44.8,57.5]$ & $0.001^{*}$ & $44.0[26.7,54.0]$ & $52.5[46.9,58.0]$ & $0.001^{*}$ \\
\hline \multicolumn{7}{|l|}{ CMR: left ventricle } \\
\hline $\begin{array}{l}\text { LV maximal wall thick- } \\
\text { ness }(\mathrm{mm})\end{array}$ & - & $19.0[16.0,22.0]$ & - & $21.0[18.0,23.0]$ & $19.0[16.0,22.0]$ & 0.068 \\
\hline
\end{tabular}


Table 1 (continued)

\begin{tabular}{|c|c|c|c|c|c|c|}
\hline & $\begin{array}{l}\text { Control subjects } \\
(n=20)\end{array}$ & $\begin{array}{l}\text { HCM population, } \\
n=238\end{array}$ & $P$ value & New onset AF ( $n=33)$ & $\begin{array}{l}\text { No new onset AF } \\
(n=205)\end{array}$ & $P$ value \\
\hline $\begin{array}{l}\text { LVOT max pressure } \\
\text { gradient }(\mathrm{mmHg})\end{array}$ & - & $5.2[3.8,7.6]$ & - & $5.4[4.0,8.1]$ & $5.2[3.8,7.4]$ & 0.698 \\
\hline $\begin{array}{l}\text { LVOT obstruc- } \\
\text { tion } \geq 30 \mathrm{mmHg}, \mathrm{n}, \%\end{array}$ & - & 9,4 & - & 0,0 & 9,4 & - \\
\hline LVEDV index $\left(\mathrm{mL} / \mathrm{m}^{2}\right)$ & $80.8[65.9,93.5]$ & $71.1[62.0,82.1]$ & 0.025 & $66.0[55.0,75.0]$ & $72.2[63.5,82.3]$ & 0.024 \\
\hline LVESV index $\left(\mathrm{mL} / \mathrm{m}^{2}\right)$ & $29.0[21.4,35.0]$ & $21.3[17.0,27.0]$ & 0.008 & $19.3[15.8,25.0]$ & $21.7[17.6,27.0]$ & 0.039 \\
\hline $\begin{array}{l}\text { LV stroke volume index } \\
\left(\mathrm{mL} / \mathrm{m}^{2}\right)\end{array}$ & $51.4[47.4,60.0]$ & $49.8[43.8,54.9]$ & 0.171 & $45.4[39.0,53.2]$ & $50.1[44.0,55.4]$ & 0.066 \\
\hline LV ejection fraction (\%) & $64.9[59.5,71.2]$ & $69.6[65.0,74.3]$ & 0.024 & $70.6(7.2)$ & $69.3(6.9)$ & 0.292 \\
\hline LV mass (grams) & $107.4[88.8,122.9]$ & $151.5[127.3,183.5]$ & $<0.001^{*}$ & $178.8[139.7,200.4]$ & $150.3[123.7,176.7]$ & 0.024 \\
\hline
\end{tabular}

Data are represented as mean \pm standard deviation, or median [IQR]

Hypothesis testing via Student's $t$-test or Mann-Whitney $U$ test, as appropriate

$A C E l$ angiotensin converting enzyme inhibitor, $A R B$ angiotensin receptor blocker, ESC European Society of Cardiology, $L A$ left atria, $L A E D V$ left atrial end diastolic volume, $L A E S V$ left atrial end systolic volume, LGE late gadolinium enhancement (5-SD), LGE \% percentage of tissue enhanced by gadolinium, $L V$ left ventricle, $L V E D V$ left ventricular end diastolic volume, LVESV left ventricular end systolic volume, LVOT left ventricular outflow tract, LVOT obstruction, when pressure $\geq 30 \mathrm{mmHg}, N Y H A$ New York Heart Association, SCD sudden cardiac death, TIA transient ischemic attack, VT ventricular tachycardia

*Indicates a significant difference. This significance level is $P<0.05$ without Bonferroni correction for baseline clinical variables and medications, whereas with Bonferroni correction the significance values are $P<0.005$ and $P<0.0071$ for CMR: Left Atrium and CMR: Left Ventricle, respectively

HCM and healthy controls even at a significant level of $P<0.05$ [17].

\section{Patients with and without new onset AF}

HCM patients who developed new onset AF were significantly older and were more likely to have mitral regurgitation, higher New York Heart Association (NYHA) scores, and non-sustained ventricular tachycardia (VT). Patients with new onset AF had higher LV wall thickness, LV mass, LAEDV index, LAESV index, and more impaired LAEF and global LA strain (reservoir, conduit, and booster) at baseline CMR than those who did not (at a $P<0.05$ level). There was a non-significant trend towards a higher LA anterior-posterior diameter at baseline in patients who developed AF versus those who did not $(P=0.136)$. Once the Bonferroni correction was applied (Table 1), only LAESV index, LAEF and global LA strain parameters remained significantly different between groups.

\section{Diagnostic accuracy of LA parameters in predicting new onset $\mathrm{AF}$ in $\mathrm{HCM}$}

The optimal threshold values for predicting new onset AF for the main LA parameters and age were calculated using ROC curve analysis. Additional file 1: Table S2 shows rounded optimal thresholds and measurements of diagnostic accuracy (C-statistic).

\section{Biomarkers associated with incident AF in HCM}

To assess the determinants of AF in HCM patients, we undertook a univariate analysis (see Table 2) and found the following variables to be significant predictors of new onset AF once Bonferroni corrections were applied: age at scan, LAESV index, LA reservoir, conduit, and booster strain, and LAEF. Of note, LA diameter (anteroposterior) assessed to be $\geq 45 \mathrm{~mm}$ on CMR (a threshold derived from transthoracic echo studies) was not significantly associated with new onset AF in our cohort even at a conventional $P$ value of $0.05(P=0.348)$.

When undertaking Cox multiple regression analysis, we considered each of the five main LA parameters that displayed evidence of significance in the Bonferroni corrected Univariate analysis. For completeness, LA diameter was also analysed in the multiple regression analysis despite not reaching significance. A model was initially constructed for each main LA parameter to avoid collinearity between them (six models shown in Table 3). Age was included in all models due to reaching significance in univariate analysis. When analysing all HCM patients, age ( $\geq 55$ years) was a significant predictor of new onset AF, with hazard ratios (HR) that ranged from 4.05 to 6.30 . LA EF $(\leq 45 \%)$ was associated with a twofold increased risk of new onset AF (Table 3). LA reservoir strain $(\leq 18 \%)$ and booster strain $(\leq 8 \%)$ were associated with a nearly threefold and fourfold increased risk of new onset AF. LAESV index, and conduit strain were not significant predictors.

Further to this, we applied the same six Cox multiple regression models to a subgroup of HCM patients in whom LA diameter was less than $45 \mathrm{~mm}(\mathrm{n}=217)$, a group that would otherwise not be considered at risk of AF based on ESC guidelines [2]. Age was again 
Table 2 Univariate predictors of new onset AF in all patients

\begin{tabular}{|c|c|c|}
\hline & \multicolumn{2}{|c|}{ Univariate analysis } \\
\hline & $\mathrm{HR}(95 \% \mathrm{Cl})$ & $\begin{array}{l}P \text { value prior } \\
\text { to correction }\end{array}$ \\
\hline \multicolumn{3}{|l|}{ Baseline and clinical } \\
\hline Age at scan, per year & $1.07(1.04-1.10)$ & $<0.001^{*}$ \\
\hline Age $\geq 55$ years (rounded $R O C$ threshold) & $5.94(2.50-14.09)$ & $<0.001^{*}$ \\
\hline Male sex & $0.95(0.43-2.11)$ & 0.903 \\
\hline Systolic blood pressure, per mmHg & $1.01(0.99-1.03)$ & 0.341 \\
\hline Diastolic blood pressure, per mmHg & $1.03(0.99-1.06)$ & 0.155 \\
\hline Body mass index, per unit & $1.03(0.96-1.12)$ & 0.393 \\
\hline Hypertension & $2.00(0.99-4.03)$ & 0.051 \\
\hline Diabetes & $0.73(0.17-3.07)$ & 0.669 \\
\hline Smoking history & $1.16(0.55-2.45)$ & 0.692 \\
\hline Hypercholesterolemia & $1.04(0.36-2.96)$ & 0.947 \\
\hline Family history of SCD 1st degree relative & $0.45(0.17-2.23)$ & 0.454 \\
\hline Family history of SCD 2 nd degree relative & $1.21(0.42-3.46)$ & 0.725 \\
\hline SCD risk score (ESC) & $1.04(0.86-1.26)$ & 0.673 \\
\hline $\mathrm{VT}$ & $1.43(0.59-3.46)$ & 0.433 \\
\hline TIA stroke & $2.92(0.69-12.31)$ & 0.145 \\
\hline Syncope & $0.48(0.11-2.02)$ & 0.318 \\
\hline NYHA class & $1.79(1.09-2.93)$ & 0.021 \\
\hline LGE, presence or absence & $6.27(0.85-46.05)$ & 0.071 \\
\hline LGE mass, g & $1.00(0.98-1.02)$ & 0.871 \\
\hline LGE, \% & $1.00(0.96-1.04)$ & 0.926 \\
\hline Presence of sarcomeric variant & $0.52(0.23-1.17)$ & 0.113 \\
\hline Mitral regurgitation presence (any grade) vs absence & $1.16(0.81-1.67)$ & 0.407 \\
\hline Mitral regurgitation grade $1 /$ nomitral regurgitation vs grade $2 / 3 / 4$ & $2.16(0.97-4.79)$ & 0.058 \\
\hline \multicolumn{3}{|l|}{ Medication } \\
\hline Beta-blockers & $1.22(0.61-2.44)$ & 0.577 \\
\hline Calcium channel blockers & $1.96(0.95-4.02)$ & 0.067 \\
\hline ACE-I/ARB & $1.61(0.78-3.34)$ & 0.201 \\
\hline Diuretics & $0.88(0.12-6.46)$ & 0.899 \\
\hline Aspirin & $1.00(0.48-2.09)$ & 0.994 \\
\hline \multicolumn{3}{|l|}{$C M R: L A$} \\
\hline LA diameter, mm, continuous & $1.05(0.99-1.12)$ & 0.046 \\
\hline LA diameter $\geq 45$ mm (ESC threshold) & $1.58(0.61-4.12)$ & 0.348 \\
\hline LA diameter $\geq 42 \mathrm{~mm}$ (ROC threshold rounded) & $2.11(1.05-4.25)$ & 0.036 \\
\hline LAEDV index, $\mathrm{mL} / \mathrm{m}^{2}$, continuous & $1.02(1.01-1.04)$ & 0.023 \\
\hline LAEDV index $\geq 50 \mathrm{~mL} / \mathrm{m}^{2}$ (ROC threshold rounded) & $2.15(1.08-4.27)$ & 0.029 \\
\hline LAESV index, $\mathrm{mL} / \mathrm{m}^{2}$, continuous & $1.04(1.02-1.06)$ & $<0.001^{*}$ \\
\hline LAESV index $\geq 27 \mathrm{~mL} / \mathrm{m}^{2}$ (ROC threshold rounded) & $2.42(1.22-4.82)$ & 0.012 \\
\hline LA reservoir strain, \% (continuous) & $0.86(0.81-0.91)$ & $<0.001^{*}$ \\
\hline LA reservoir strain $\leq 18 \%$ (ROC threshold rounded) & $3.64(1.81-7.32)$ & $<0.001^{*}$ \\
\hline LA conduit strain, $\%$, continuous & $0.88(0.81-0.95)$ & $0.002^{*}$ \\
\hline LA conduit strain $\leq 12 \%(\mathrm{ROC}$ threshold rounded $)$ & $3.15(1.29-7.72)$ & 0.012 \\
\hline LA booster strain, \%, continuous & $0.87(0.78-0.97)$ & 0.014 \\
\hline LA booster strain $\leq 8 \%$ (ROC threshold rounded) & $2.93(1.44-5.95)$ & $0.003^{*}$ \\
\hline LAEF (\%) continuous & $0.94(0.92-0.97)$ & $<0.001^{*}$ \\
\hline LAEF $\leq 45 \%$, (ROC threshold rounded) & $3.42(1.72-6.78)$ & $<0.001^{*}$ \\
\hline
\end{tabular}


Table 2 (continued)

\section{Univariate analysis}

HR $(95 \% \mathrm{Cl}) \quad$ P value prior

to correction

CMR: left ventricle

LV maximal wall thickness, $\mathrm{mm}$

LV mass, grams

LVOT max pressure gradient, $\mathrm{mmHg}$

LVEDV index $\left(\mathrm{mL} / \mathrm{m}^{2}\right)$

LVESV index $\left(\mathrm{mL} / \mathrm{m}^{2}\right)$

LV stroke volume index, $\mathrm{mL} / \mathrm{m}^{2}$

LV ejection fraction, \%

$\begin{array}{ll}1.06(1.00-1.14) & 0.059 \\ 1.00(1.00-1.01) & 0.180 \\ 1.04(0.95-1.14) & 0.350 \\ 0.98(0.95-1.00) & 0.069 \\ 0.96(0.91-1.01) & 0.114 \\ 0.98(0.94-1.01) & 0.163 \\ 1.02(0.97-1.07) & 0.506\end{array}$

LA left atria, LAEDV left atrial end diastolic volume, $L A E F$ left atrial ejection fraction, $L A E S V$ left atrial end systolic volume, $L G E$ late gadolinium enhancement (5-SD), $L G E$ $\%$ percentage of tissue enhanced by gadolinium, LV left ventricle, LVOT left ventricular outflow tract, LVEDV left ventricular end diastolic volume, LVESV left ventricular end systolic volume, LVSV left ventricular stroke volume, NYHA New York Heart Association, SCD sudden cardiac death, TIA transient ischemic attack, VT ventricular tachycardia

*Indicates a significant difference given Bonferroni correction to the $P$ value as stated in the methods

Table 3 Six multiple regression models: determinants of AF in all HCM patients, and in those with LA diameter $<45 \mathrm{~mm}$, indicating a low risk for AF development

\begin{tabular}{|c|c|c|c|c|c|c|}
\hline & & & Model 3 & Model 4 & Model 5 & Model 6 \\
\hline & LA diameter & LAESV index & LA reservoir strain & LA conduit strain & LA booster strain & LAEF \\
\hline & $\mathrm{HR}(95 \% \mathrm{Cl})$ & $\mathrm{HR}(95 \% \mathrm{Cl})$ & $\mathrm{HR}(95 \% \mathrm{Cl})$ & $\mathrm{HR}(95 \% \mathrm{Cl})$ & $\mathrm{HR}(95 \% \mathrm{Cl})$ & $\mathrm{HR}(95 \% \mathrm{Cl})$ \\
\hline \multicolumn{7}{|l|}{ All HCM patients, $n=238$} \\
\hline Age $\geq 55$ & $5.07(2.23-11.55)^{* *}$ & $4.72(2.06-10.95)^{* *}$ & $4.05(1.73-9.46)^{* *}$ & $4.42(1.76-11.06)^{* *}$ & $6.62(2.79-15.70)^{* *}$ & $4.19(1.80-9.76)^{* *}$ \\
\hline LA diameter $\geq 42$ mm & $1.92(0.95-3.91)$ & - & - & - & - & - \\
\hline LAESV inde $x \geq 27 \mathrm{~mL} / \mathrm{m}^{2}$ & - & $1.82(0.90-3.68)$ & - & - & - & - \\
\hline LA reservoir strain $\leq 18 \%$ & - & - & $2.56(1.24-5.27)^{*}$ & - & - & - \\
\hline LA conduit strain $\leq 12 \%$ & - & - & - & $1.58(0.58-4.25)$ & - & - \\
\hline LA booster strain $\leq 8 \%$ & - & - & - & - & $3.69(1.81-7.52)^{* *}$ & - \\
\hline $\mathrm{LAEF} \leq 45 \%$ & - & - & - & - & - & $2.43(1.20-4.92)^{*}$ \\
\hline \multicolumn{7}{|l|}{$\begin{array}{l}\text { HCM patients with LA } \\
\text { diameter }<45 \mathrm{~mm} \text {, } \\
\mathrm{n}=217\end{array}$} \\
\hline Age $\geq 55$ & $4.05(1.72-9.51)^{* *}$ & $4.73(2.04-10.96)^{* *}$ & $3.34(1.38-8.06)^{* *}$ & $3.41(1.30-8.96)^{*}$ & $5.78(2.35-14.23)^{* *}$ & $3.67(1.53-8.81)^{* *}$ \\
\hline LA diameter $\geq 42 \mathrm{~mm}$ & $1.94(0.84-4.47)$ & - & - & - & - & - \\
\hline LAESV index $\geq \mathrm{mL} / \mathrm{m}^{2}$ & - & $1.82(0.90-3.68)$ & - & - & - & - \\
\hline LA reservoir strain $\leq 18 \%$ & - & - & $2.49(1.13-5.32)^{*}$ & - & - & - \\
\hline LA conduit strain $\leq 12 \%$ & - & - & - & $1.72(0.60-4.90)$ & - & - \\
\hline LA booster strain $\leq 8 \%$ & - & - & - & - & $3.74(1.70-8.07)^{* *}$ & - \\
\hline$L A E F \leq 45 \%$ & - & - & - & - & - & $1.98(0.92-4.28)$ \\
\hline
\end{tabular}

$E F$, ejection fraction, $H C M$, hypertrophic cardiomyopathy, $H R$, hazard ratio, $L A$, left atria, $L A E D V$, left atrial end diastolic volume, $L A E F$, left atrial ejection fraction *Indicates a significant difference of $\mathrm{P}<0.05$

**Indicates a significant difference of $P<0.01$

a significant predictor of AF in each model. Of the LA parameters, LA reservoir (HR 2.49, CI 1.13-5.32, $P=0.023$ ) and booster (HR 3.74, CI 1.70-8.07, $P \leq 0.001$ ) strain were the only variables to retain significance (Table 3).
We then included all the parameters that retained significance in the Cox multiple regression models on all HCM patients in a model (Table 4). In this, only age and LA booster function retained significance, with age 
Table 4 Multiple regression: predicting new onset AF in all HCM patients

\begin{tabular}{llc}
\hline & HR $(\mathbf{9 5} \% \mathbf{C l})$ & P value \\
\hline Age $\geq 55$ years & $5.22(2.12-12.83)$ & $<0.001^{* *}$ \\
LA reservoir strain $\leq 18 \%$ & $1.21(0.44-3.32)$ & 0.717 \\
LA booster strain $\leq 8 \%$ & $3.08(1.43-6.64)$ & $0.004^{* *}$ \\
LAEF $\leq 45 \%$ & $1.73(0.68-4.40)$ & 0.252 \\
\hline
\end{tabular}

LAEF left atrial ejection fraction, $H R$ hazard ratio, $L A$ left atria, $L A E D V$ left atrial end diastolic volume

*Indicates a significant difference of $\mathrm{P}<0.05$

**Indicates a significant difference of $P<0.01$

being a more powerful predictor than booster strain (HR of 5.22 vs 3.08).

\section{Survival analysis}

Survival graphs displaying survival free from AF were produced for the variables that reached significance in the multiple regression models (Fig. 2). There was a significant difference in freedom from AF in the 10 years post follow up when stratifying by LA diameter, LAEDV index, LAEF, and in particular age. When all the HCM cases were stratified by an LA reservoir strain of $18 \%$, the 5 years survival free of AF was $76 \%$ for those with reservoir strain $\leq 18 \%$, compared to $96 \%$ in those with strain $>18 \%$. Similarly, when stratifying with LA booster strain of $8 \%$, the 5 years survival free of AF was $81 \%$ in those with booster strain $\leq 8 \%$, compared to $94 \%$ in those with booster strain $>8 \%$.

\section{Determinants of LA strain components}

Univariate and multivariable determinants of LA reservoir, conduit, and booster strain are shown in Table 5. On multivariable regression analysis, age at scan associated with all three of reservoir, conduit, and booster strain. In addition, NYHA class, LV maximal wall thickness, LV mass, and LV ejection fraction (LVEF) also remained significantly associated with reservoir strain. Presence of

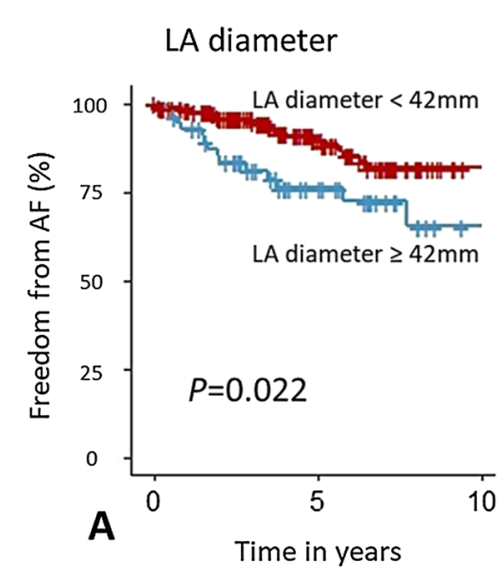

LA volume indexed

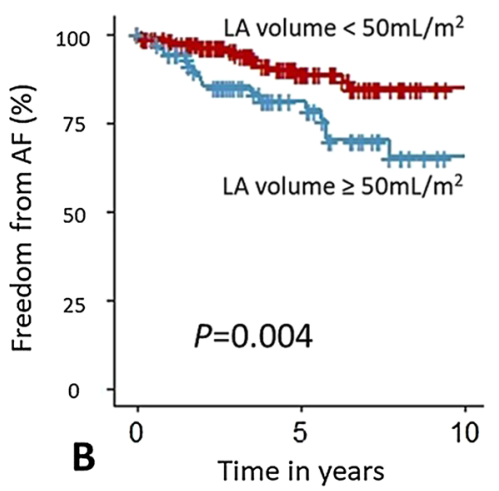

LA ejection fraction

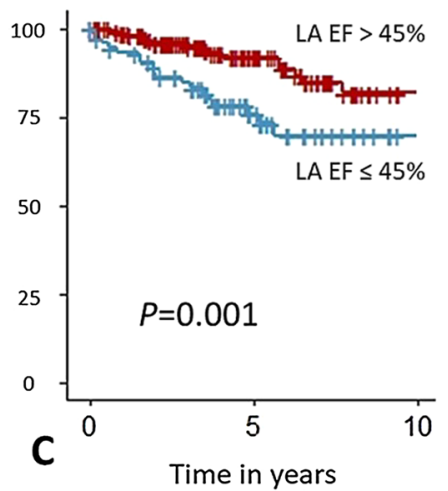

Age

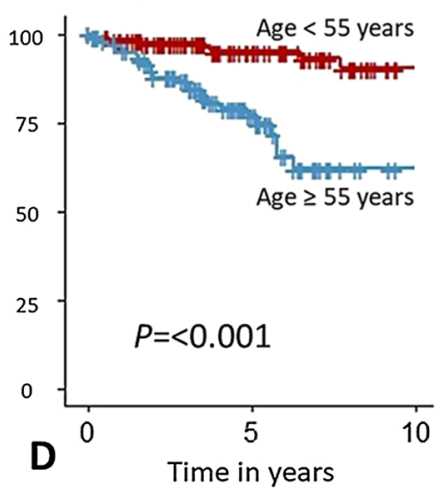

\section{LA reservoir strain}

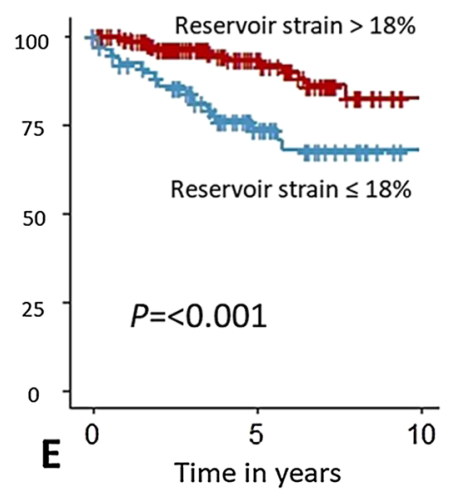

LA booster strain

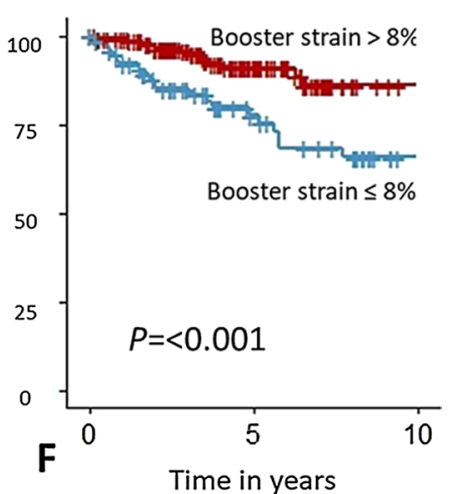

Fig. 2 Kaplan Meier curves demonstrating freedom from atrial fibrillation for HCM patients. Survival curves of all HCM patients $(n=238)$ showing freedom from atrial fibrillation (AF) when stratified according to the variables included in the Cox multiple regression analysis: $\mathbf{A}$ Left atrial (LA) diameter; $\mathbf{B} L A$ end diastolic volume (LAEDV) index; C LA ejection fraction; $\mathbf{D}$ Age ; E LA reservoir strain; $\mathbf{F} L A$ booster strain 


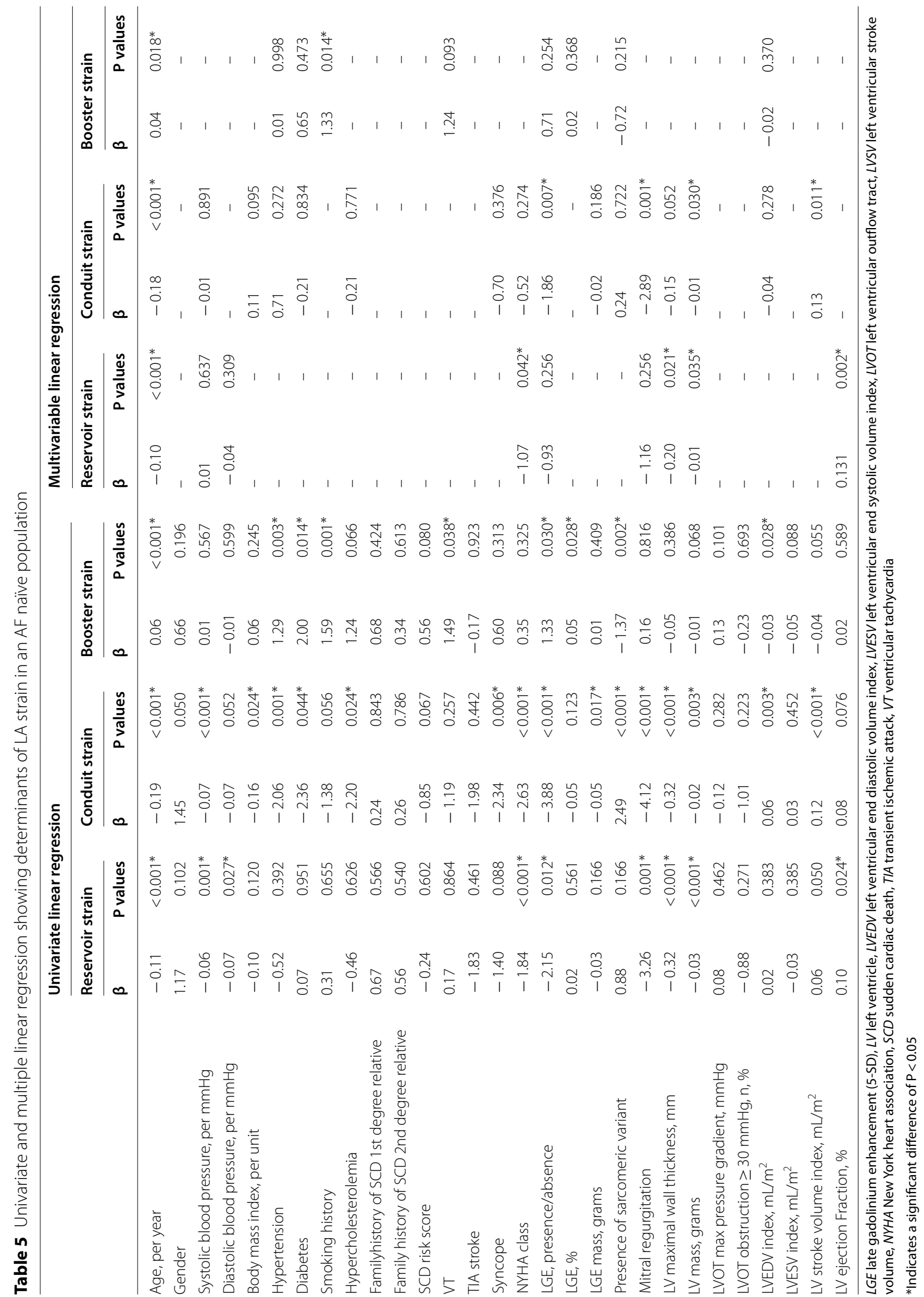


LGE, mitral regurgitation, LV mass, and LV stroke volume also independently associated with conduit strain, whilst a history of smoking also associated with booster strain.

\section{Discussion}

In this study, we used CMR to assess the role of LA deformation when compared to standard LA parameters and baseline characteristics in predicting new onset AF in HCM. In addition to age and LAEF, we have shown that LA reservoir and booster strain have the ability to augment prediction of new onset AF in HCM. Specifically, we show that LA reservoir and booster strain dysfunction are important determinants of AF risk even in those with an LA diameter less than $45 \mathrm{~mm}$, a threshold set by the ESC to escalate arrhythmia surveillance frequency. Of interest, a marginally higher risk of incident AF was specifically seen in those with booster strain dysfunction compared to other parameters. We also determined the factors that associate with markers of atrial deformation and show that age and phenotypic severity adversely influenced LA deformation.

The prediction of new onset AF in HCM allows for intensification of monitoring and prophylactic use of anticoagulation in those at high risk, thus minimising the effects of subsequent harmful sequelae, primarily stroke, myocardial infarction (embolic), and heart failure [24]. Early studies investigating the determinants of new onset AF have found LA size to be important in guiding surveillance. More recently, LA function has emerged as a useful determinant of new onset AF in HCM patients [4-7]. LA deformation or strain provides an additional measure of atrial mechanics and has been found to provide deeper insights into the risk of arrhythmia, embolic events, and other adverse outcomes in some cardiovascular diseases [25, 26]. In patients with AF, Hsu et al. demonstrated an independent association between LA strain and risk of embolic complications such as stroke [27].

Most studies to date have used transthoracic echocardiography (TTE) for the assessment of LA strain in HCM $[14,15]$. However, TTE remains limited in its ability to provide consistently high quality images which are often confounded by body habitus, comorbidities such as chronic obstructive lung disease, and operator expertise [28]. In comparison, CMR provides an excellent platform to precisely and reproducibly evaluate cardiac chambers, providing additional information on myocardial tissue characteristics [29]. The high resolution images acquired by CMR enable retrospective analyses of LA dimensions and function from both LA and right atria, which are well visualised in standard cardiac planes acquired in accordance with consensus guidelines [30]. In spite of the increasing use of CMR for assessment of HCM patients, studies examining the additive value of LA strain assessment on CMR in AF prediction in HCM are lacking.

Here, we systematically examined components of LA strain as assessed on CMR, in addition to standard metrics of LA size and function. Consistent with previous studies, we found LA reservoir and conduit strain to be impaired in HCM patients [13, 31], though after correcting for multiple comparisons only conduit strain remained impaired. By contrast, LA booster strain, the active component of LA deformation, was not significantly different between HCM patients and healthy control subjects. Indeed, the evidence in support of booster dysfunction in HCM patients is conflicting. In a study by Yang and colleagues [17], LA booster strain was not different between non-obstructive HCM patients and controls. In contrast, Kowallick et al. reported a significant impairment in LA booster strain in HCM patients relative to controls [32].

A number of studies have postulated that booster function could indicate an increase in fibrotic burden of the atria [33, 34]. In a recent study by Sivalokanathan et al. [35], atrial fibrosis as detected by atrial LGE was found to be greater in those with new onset AF [35]. Likewise, in our study booster strain was reduced in those patients that developed AF compared to those that did not. Thus, the relationship between booster strain and AF may potentially reflect an increased burden of atrial fibrosis or an underlying atrial myopathy in HCM patients.

The absolute values of LA strain reported in this study were lower than those reported elsewhere [36], though comparable with some studies using CMR myocardial feature tracking. Values from healthy, yet elderly controls in studies by Evin et al. [37] and Lamy et al. [38] matched our relatively old and obese control group closely [37, 38]. The values from all HCM patients in this study are within reasonable range of those presented by Sivalokanathan et al. and Kowallick $[13,35]$. In the present study, LA diameter [5], volume [6] and LAEF [7] were univariate determinants of AF risk, largely consistent with earlier work. We additionally showed that LA reservoir and booster strain are associated with a threefold and fourfold increase in new onset AF risk, respectively, and, as illustrated by the freedom-from-AF survival curves (see Fig. 2 and Additional file 1: Fig. S2), both measures discriminated risk of new onset AF with reasonable diagnostic accuracies (see Additional file 1: Table S2). Of importance, in patients with LA diameter of less than $45 \mathrm{~mm}$, booster and reservoir strain remained strong independent determinants of new onset AF.

To date, there have been a number of studies of HCM patients in which age independently predicted AF risk [4, 24, 39]. Concordantly, we also found age to be a strong determinant of future risk of new onset AF. 
Consensus guidelines make no specific recommendations for prophylactic anticoagulation or frequency of arrhythmia monitoring in patients based on age. Our findings highlight the need to incorporate age into future risk prediction models being developed for AF or stroke risk prediction in patients with HCM and provide an argument to consider prophylactic anticoagulation therapy in elderly HCM patients who are not at risk of significant bleeding.

Wider evidence surrounding the impact of sarcomeric mutations on AF incidence is mixed; Bongini et al., found no association between HCM genetic subtype and AF [40], whereas Lee et al. report myosin heavy chain-7 (MYH7) to be predictive of AF [41]. In this study, the presence of sarcomeric variant trended towards associating with lower risk of AF on univariate analysis (HR of $0.52, P=0.113$ ). We believe that this may have been due to a higher prevalence of hypertension and increased age among the sarcomere negative HCM patients (Additional file 1: Table S3). Such differences in baseline characteristics of HCM patients with and without sarcomeric variants have also been observed in a recent multicentre CMR registry of HCM (HCMR study) [42].

This study assessed the determinants of LA strain in an AF naïve HCM population. Our findings in the multiple regression models suggest that age, markers of phenotypic severity (LV wall thickness, mass, mitral regurgitation, LGE) and baseline SCD risk are associated with adverse LA mechanical remodelling and could explain the emerging prognostic implications of LA strain for many cardiovascular events $[11,14,26]$. That age was negatively associated with reservoir and conduit strain and positively associated with booster strain has been noted previously in non-HCM populations-atrial emptying is more dependent on booster function in older age $[43,44]$. The negative correlation between LGE and conduit strain and LV mass/ wall thickness and reservoir strain in HCM is also not surprising given that both conduit and reservoir largely reflect LA compliance, which may be impacted by the burden of fibrosis and adverse remodelling [45]. Although the presence of sarcomeric variant was seen to associate with both conduit and booster strain on univariate analysis, this failed to reach statistical significance in our multivariable model. This may be because of the differences in baseline characteristics (in particular age and hypertension) of sarcomere positive and negative HCM patients. Further studies are needed to tease out the precise relationship between sarcomeric variant status and LA strain components.

\section{Limitations}

This is a retrospective single-centre study, and thus some characteristics, such as the extent of LVOT obstruction, are not reflective of the population frequency. Temporal resolution of cine imaging was not consistent (39-60 ms) in all patients (due to the retrospective nature of this study) and could in theory contribute to variability in strain measurements. Prior to the publication of ESC guidelines in 2014, TTE analysis was only done as per clinical need. Therefore, we lacked the ability to compare or correlate CMR and TTE data. Similarly, diastolic function was not available on the same day as CMR for many patients. A dedicated atrial stack on LGE imaging was not undertaken and therefore fibrosis in the atria could not be assessed. The frequency of AF monitoring is in line with current clinical practice. We did not assess atrial strain rate as this was beyond the remit of our primary hypothesis.

The LA strain values of controls presented in this paper differ from some of the wider literature. However, our healthy controls were older, obese, and matched to HCM cohort (for blood pressure) and thus different to controls from other studies. These factors may explain potential differences in magnitude of strain. Another reason for differences CMR strain measurements across studies is the lack of standardisation in strain measurements across different analysis platforms. The increasing use of fully automated machine learning algorithms is expected to significantly improve standardisation and reduce interobserver variability, at which point CMR analysis of the LA strain could become a valuable clinically tool for risk prediction of AF. Finally, we did not undertake external validation of the thresholds for LA parameters and age, hence the selected thresholds require cautious interpretation when extrapolating to all HCM patients. Nevertheless, the present study provides novel insights into the additional value of LA deformation analysis on CMR for stratifying HCM patients at risk of new onset AF.

\section{Conclusion}

We used CMR in HCM and demonstrate that LA strain components are reduced in patients who develop AF. Specifically, reservoir and booster LA strain augment risk prediction of new onset AF in HCM patients. Our work underscores the importance of age as a guide to arrhythmia surveillance and suggests that the routine assessment of LA strain in HCM patients could augment existing tools for AF and potentially stroke prediction in HCM. 


\begin{abstract}
Abbreviations
AF: Atrial fibrillation; AHA: American Heart Association; AUC: Area under curve; BMI: Body mass index; CMR: Cardiovascular magnetic resonance; $\mathrm{Cl}$ : Confidence interval; ECG: Electrocardiogram; ESC: European Society of Cardiology; EF: Ejection fraction; HCM: Hypertrophic cardiomyopathy; HR: Hazard ratio(s); ICC: Inherited Cardiac Conditions; LA: Left atrium/left atrial; LAEDV: Left atrial end-diastolic volume; LAEF: Left atrial ejection fraction; LAESV: Left atria end-systolic volume; LASV: Left atrial stroke volume; LGE: Late gadolinium enhancement; LV: Left ventricle/left ventricular; LVEDV: Left ventricular enddiastolic volume; LVESV: Left ventricular end-systolic volume; LVOT: Left ventricular outflow tract; NPV: Negative predictive value; NYHA: New York Heart Association; PPV: Positive predictive value; ROC: Receiver Operator Characteristic; SCD: Sudden cardiac death; TTE: Transthoracic echocardiography; VUS: Variant of uncertain significance; $\mathrm{VT}$ : Ventricular tachycardia.
\end{abstract}

\section{Supplementary Information}

The online version contains supplementary material available at https://doi. org/10.1186/s12968-021-00793-6.

Additional file 1: Table S1. Reproducibility of strain parameters.

Table S2. Diagnostic accuracy of predicting new onset AF. Table S3. Differences in age and rate of hypertension between those with and without sarcomeric variants. Figure S1. Flow chart showing inclusion of subjects. Figure S2. Survival curve for HCM patients.

\section{Acknowledgements}

Not applicable.

\section{Authors' contributions}

BR contributed to ethics, undertook scanning of some patients, conceived the study, drafted the manuscript. RS performed image analysis, statistical analysis, and drafted the manuscript. MM, KC, CN, GT, FR contributed to data entry and image analysis. $\mathrm{EO}$ and RA contributed to ethics and recruitment of patient. $K T, A L, A H, E C W, B C$ drafted the manuscript. HW and SN conceived the study and contributed to manuscript drafting and preparation. All authors read and approved the final manuscript.

\section{Funding}

This study was funded by the National Institute of Health Research (NIHR) Oxford Biomedical Research Centre and the British Heart Foundation. B.R and M.M were funded by the Oxford British Heart Foundation Centre of Research Excellence (RE/18/3/34214) and National Institute of Health Research Oxford Biomedical Research Centre. R.A. was funded by a British Heart Foundation Clinical Research Training Fellowship Grant (RA: 098436/Z/12/Z). SN and HW acknowledge support from the Oxford British Heart Foundation Centre of Research Excellence. BC acknowledges the support of the NIHR Oxford Biomedical Research Centre and the British Heart Foundation.

\section{Availability of data and materials}

The datasets used and/or analysed during the current study are available from the corresponding author on reasonable request.

\section{Declarations}

\section{Ethics approval and consent to participate}

This study was approved by the local Oxford ethics committee (Reference: 07/ Q1607/66, 12/LO/ 1979). All patient included consent for their details to be used for research purposes.

\section{Consent for publication}

Not applicable.

\section{Competing interests}

$\mathrm{SN}$ reports a consultancy and a grant from Cytokinetics, as well as grant support from Boehringer Ingelheim.

\section{Author details}

${ }^{1}$ University of Oxford Centre for Clinical Magnetic Resonance Research (OCMR), Division of Cardiovascular Medicine, Radcliffe Department of Medicine, University of Oxford, Oxfordshire OX3 9DU, United Kingdom. ²Division of Cardiovascular Medicine, NIHR Oxford Biomedical Research Centre, University of Oxford, Oxford, UK.

Received: 18 January 2021 Accepted: 8 July 2021

Published online: 11 October 2021

\section{References}

1. Siontis KC, Geske JB, Ong K, Nishimura RA, Ommen SR, Gersh BJ. Atrial fibrillation in hypertrophic cardiomyopathy: prevalence, clinical correlations, and mortality in a large high-risk population. J Am Heart Assoc. 2014;3(3):e001002.

2. Authors/Task Force m, Elliott PM, Anastasakis A, Borger MA, Borggrefe M, Cecchi F, et al. 2014 ESC Guidelines on diagnosis and management of hypertrophic cardiomyopathy: the Task Force for the Diagnosis and Management of Hypertrophic Cardiomyopathy of the European Society of Cardiology (ESC). Eur Heart J. 2014;35(39):2733-79.

3. Schaer BA, Zellweger MJ, Cron TA, Kaiser CA, Osswald S. Value of routine Holter monitoring for the detection of paroxysmal atrial fibrillation in patients with cerebral ischemic events. Stroke. 2004;35(3):e68-70.

4. Maron BJ, Haas TS, Maron MS, Lesser JR, Browning JA, Chan RH, et al. Left atrial remodeling in hypertrophic cardiomyopathy and susceptibility markers for atrial fibrillation identified by cardiovascular magnetic resonance. Am J Cardiol. 2014;113(8):1394-400.

5. Guttmann OP, Rahman MS, O'Mahony C, Anastasakis A, Elliott PM. Atrial fibrillation and thromboembolism in patients with hypertrophic cardiomyopathy: systematic review. Heart. 2014;100(6):465-72.

6. Yang WI, Shim CY, Kim YJ, Kim SA, Rhee SJ, Choi EY, et al. Left atrial volume index: a predictor of adverse outcome in patients with hypertrophic cardiomyopathy. J Am Soc Echocardiogr. 2009;22(12):1338-43.

7. Debonnaire P, Joyce E, Hiemstra Y, Mertens BJ, Atsma DE, Schalij MJ, et al. Left atrial size and function in hypertrophic cardiomyopathy patients and risk of new-onset atrial fibrillation. Circ Arrhythmia Electrophysiol. 2017. https://doi.org/10.1161/CIRCEP.116.004052.

8. Gersh BJ, Maron BJ, Bonow RO, Dearani JA, Fifer MA, Link MS, et al. 2011 ACCF/AHA Guideline for the Diagnosis and Treatment of Hypertrophic Cardiomyopathy: a report of the American College of Cardiology Foundation/American Heart Association Task Force on Practice Guidelines. Developed in collaboration with the American Association for Thoracic Surgery, American Society of Echocardiography, American Society of Nuclear Cardiology, Heart Failure Society of America, Heart Rhythm Society, Society for Cardiovascular Angiography and Interventions, and Society of Thoracic Surgeons. J Am Coll Cardiol. 2011;58(25):e212-60.

9. Williams LK, Chan RH, Carasso S, Durand M, Misurka J, Crean AM, et al. Effect of left ventricular outflow tract obstruction on left atrial mechanics in hypertrophic cardiomyopathy. BioMed Res Int. 2015. https://doi.org/ 10.1155/2015/481245.

10. Huang $X$, Yue $Y$, Wang $Y$, Deng $Y$, Liu L, Di Y, et al. Assessment of left ventricular systolic and diastolic abnormalities in patients with hypertrophic cardiomyopathy using real-time three-dimensional echocardiography and two-dimensional speckle tracking imaging. Cardiovasc Ultrasound. 2018;16(1):23.

11. Gan GCH, Ferkh A, Boyd A, Thomas L. Left atrial function: evaluation by strain analysis. Cardiovasc Diagn Ther. 2018;8(1):29-46.

12. To AC, Flamm SD, Marwick TH, Klein AL. Clinical utility of multimodality LA imaging: assessment of size, function, and structure. JACC CardiovasC Imaging. 2011;4(7):788-98.

13. Kowallick JT, Kutty S, Edelmann F, Chiribiri A, Villa A, Steinmetz M, et al. Quantification of left atrial strain and strain rate using Cardiovascular Magnetic Resonance myocardial feature tracking: a feasibility study. J Cardiovasc Magn Reson. 2014;16:60.

14. Essayagh B, Resseguier N, Michel N, Casalta A-C, Renard S, Donghi V, et al. Left atrial dysfunction as marker of poor outcome in patients with hypertrophic cardiomyopathy. Arch Cardiovasc Dis. 2021;114(2):96-104. 
15. Vasquez N, Ostrander BT, Lu D-Y, Ventoulis I, Haileselassie B, Goyal S, et al. Low left atrial strain is associated with adverse outcomes in hypertrophic cardiomyopathy patients. J Am Soc Echocardiogr. 2019;32(5):593-603.e1.

16. Kim K-J, Choi H-M, Yoon YE, Kim H-L, Lee S-P, Kim H-K, et al. Left atrial mechanical function and global strain in hypertrophic cardiomyopathy. PLOS ONE. 2016;11(6):e0157433.

17. Yang $Y$, Yin G, Jiang $Y$, Song L, Zhao S, Lu M. Quantification of left atrial function in patients with non-obstructive hypertrophic cardiomyopathy by cardiovascular magnetic resonance feature tracking imaging: a feasibility and reproducibility study. J Cardiovasc Magn Reson. 2020;22(1):1-11.

18. Ariga R, Tunnicliffe EM, Manohar SG, Mahmod M, Raman B, Piechnik SK, et al. Identification of myocardial disarray in patients with hypertrophic cardiomyopathy and ventricular arrhythmias. J Am Coll Cardiol. 2019;73(20):2493-502.

19. Li R, Yang Z-G, Xu H-Y, Shi K, Liu X, Diao K-Y, et al. Myocardial deformation in cardiac amyloid light-chain amyloidosis: assessed with 3t cardiovascular magnetic resonance feature tracking. Sci Rep. 2017. https://doi.org/10. 1038/s41598-017-03699-5.

20. Raman B, Ariga R, Spartera M, Sivalokanathan S, Chan K, Dass S, et al. Progression of myocardial fibrosis in hypertrophic cardiomyopathy: mechanisms and clinical implications. Eur Heart J Cardiovasc Imaging. 2019;20(2):157-67.

21. Chirinos JA, Sardana M, Ansari B, Satija V, Kuriakose D, Edelstein I, et al. Left atrial phasic function by cardiac magnetic resonance feature tracking is a strong predictor of incident cardiovascular events. Circ Cardiovasc Imaging. 2018;11(12):e007512

22. Chirinos JA, Sardana M, Ansari B, Satija V, Kuriakose D, Edelstein I, et al. Left atrial phasic function by cardiac magnetic resonance feature tracking is a strong predictor of incident cardiovascular events. Circ Cardiovasc Imaging. 2018;11(12):e007512.

23. Armstrong RA. When to use the Bonferroni correction. Ophthalmic Physiol Opt. 2014;34(5):502-8.

24. Olivotto I, Cecchi F, Casey SA, Dolara A, Traverse JH, Maron BJ. Impact of atrial fibrillation on the clinical course of hypertrophic cardiomyopathy. Circulation. 2001;104(21):2517-24

25. Fujimoto K, Inoue K, Saito M, Higashi H, Kono T, Uetani T, et al. Incremental value of left atrial active function measured by speckle tracking echocardiography in patients with hypertrophic cardiomyopathy. Echocardiography. 2018;35(8):1138-48.

26. Modin D, Biering-Sorensen SR, Mogelvang R, Alhakak AS, Jensen JS, Biering-Sorensen T. Prognostic value of left atrial strain in predicting cardiovascular morbidity and mortality in the general population. Eur Heart J Cardiovasc Imaging. 2019;20(7):804-15.

27. Hsu PC, Lee WH, Chu CY, Lee HH, Lee CS, Yen HW, et al. Prognostic role of left atrial strain and its combination index with transmitral E-wave velocity in patients with atrial fibrillation. Sci Rep. 2016;6:17318.

28. Brenes JC, Doltra A, Prat S. Cardiac magnetic resonance imaging in the evaluation of patients with hypertrophic cardiomyopathy. Glob Cardiol Sci Pract. 2018;2018(3):22

29. Zareian M, Ciuffo L, Habibi M, Opdahl A, Chamera EH, Wu CO, et al. Left atrial structure and functional quantitation using cardiovascular magnetic resonance and multimodality tissue tracking: validation and reproducibility assessment. J Cardiovasc Magn Reson. 2015;17:52.

30. Hundley WG, Bluemke D, Bogaert JG, Friedrich MG, Higgins CB, Lawson MA, et al. Society for Cardiovascular Magnetic Resonance guidelines for reporting cardiovascular magnetic resonance examinations. J Cardiovasc Magn Reson. 2009;11(1):5.
31. Hinojar R, Zamorano JL, Fernandez-Mendez M, Esteban A, Plaza-Martin M, Gonzalez-Gomez A, et al. Prognostic value of left atrial function by cardiovascular magnetic resonance feature tracking in hypertrophic cardiomyopathy. Int J Cardiovasc Imaging. 2019;35(6):1055-65.

32. Kowallick JT, Silva Vieira M, Kutty S, Lotz J, Hasenfu G, Chiribiri A, et al. Left atrial performance in the course of hypertrophic cardiomyopathy: relation to left ventricular hypertrophy and fibrosis. Invest Radiol. 2017; 52(3):177-85.

33. Habibi M, Lima JA, Khurram IM, Zimmerman SL, Zipunnikov V, Fukumoto $K$, et al. Association of left atrial function and left atrial enhancement in patients with atrial fibrillation: cardiac magnetic resonance study. Circ Cardiovasc Imaging. 2015;8(2):e002769.

34. Gasparovic H, Cikes M, Kopjar T, Hlupic L, Velagic V, Milicic D, et al. Atrial apoptosis and fibrosis adversely affect atrial conduit, reservoir and contractile functions. Interact Cardiovasc Thorac Surg. 2014;19(2):223-30; discussion 30.

35. Sivalokanathan S, Zghaib T, Greenland GV, Vasquez N, Kudchadkar SM, Kontari E, Lu DY, Dolores-Cerna K, van der Geest RJ, Kamel IR, Olgin JE. Hypertrophic cardiomyopathy patients with paroxysmal atrial fibrillation have a high burden of left atrial fibrosis by cardiac magnetic resonance imaging. JACC Clin Electrophysiol. 2019;5(3):364-75.

36. Truong VT, Palmer C, Wolking S, Sheets B, Young M, Ngo TNM, et al. Normal left atrial strain and strain rate using cardiac magnetic resonance feature tracking in healthy volunteers. Eur Heart J Cardiovasc Imaging. 2020;21(4):446-53.

37. Evin M, Cluzel P, Lamy J, Rosenbaum D, Kusmia S, Defrance C, et al. Assessment of left atrial function by MRI myocardial feature tracking. J Magn Reson Imaging. 2015;42(2):379-89.

38. Lamy J, Soulat G, Evin M, Huber A, de Cesare A, Giron A, et al. Scan-rescan reproducibility of ventricular and atrial MRI feature tracking strain. Comput Biol Med. 2018:92:197-203.

39. Losi MA, Betocchi S, Aversa M, Lombardi R, Miranda M, D’Alessandro G, et al. Determinants of atrial fibrillation development in patients with hypertrophic cardiomyopathy. Am J Cardiol. 2004;94(7):895-900.

40. Bongini C, Ferrantini C, Girolami F, Coppini R, Arretini A, Targetti M, et al. Impact of genotype on the occurrence of atrial fibrillation in patients with hypertrophic cardiomyopathy. Am J Cardiol. 2016;117(7):1151-9.

41. Lee SP, Ashley EA, Homburger J, Caleshu C, Green EM, Jacoby D, et al. Incident atrial fibrillation is associated with myh7 sarcomeric gene variation in hypertrophic cardiomyopathy. Circ Heart Fail. 2018;11(9):e005191.

42. Neubauer S, Kolm P, Ho CY, Kwong RY, Desai MY, Dolman SF, et al. Distinct subgroups in hypertrophic cardiomyopathy in the NHLBI HCM Registry. J Am Coll Cardiol. 2019;74(19):2333-45.

43. Spencer KT, Mor-Avi V, Gorcsan J 3rd, DeMaria AN, Kimball TR, Monaghan MJ, et al. Effects of aging on left atrial reservoir, conduit, and booster pump function: a multi-institution acoustic quantification study. Heart (British Cardiac Society). 2001;85(3):272-7.

44. Maceira AM, Cosin-Sales J, Prasad SK, Pennell DJ. Characterization of left and right atrial function in healthy volunteers by cardiovascular magnetic resonance. J Cardiovasc Magn Reson. 2016;18(1):64.

45. Weil BR, Techiryan G, Suzuki G, Konecny F, Canty JM. Adaptive reductions in left ventricular diastolic compliance protect the heart from stretchinduced stunning. JACC Basic TransI Sci. 2019:4:527-41.

\section{Publisher's Note}

Springer Nature remains neutral with regard to jurisdictional claims in published maps and institutional affiliations. 